\title{
ASCO-Highlights 2016
}

Anfang Juni 2016 fand wieder der weltgrößte Krebskongress statt, das Jahresmeeting der American Society of Clinical Oncology (ASCO). 35000 Teilnehmer aus aller Welt informierten sich über aktuelle Studiendaten zu einem breiten Spektrum von Tumorentitäten. Entsprechend dem Kongressmotto 'Collective Wisdom - the Future of Patient-Centered Research' hatte in diesem Jahr auch die Supportivtherapie einen hohen Stellenwert. Insgesamt gab es zwei große Trends beim ASCO, die schon in der Vorjahren im Fokus standen, sich aber nun deutlich verfestigt und weiterentwickelt haben: die immer präziser werdende zielgerichtete Therapie und die Immuntherapie. Hierzu wurden neue Studien und Langzeitdaten vorgestellt. Zudem geht die Suche nach Biomarkern zur Identifikation der optimalen Patientenpopulationen für zielgerichtete Therapien und Immuntherapien weiter.

Alle Highlights des ASCO in einem Artikel zusammenzufassen ist nicht möglich. Deshalb sollen hier - unabhängig von den allgemeinen Trends Präzisonsmedizin und Immuntherapie - einige ausgewählte Studien mit klinischer Relevanz vorgestellt werden, die bei den Experten vor Ort Aufsehen erregten.

\section{Mammakarzinom: 10 Jahre Aromatasehemmung neuer Behandlungsstandard?}

Nachdem bereits vor einigen Jahren die ATLAS-Studie gezeigt hatte, dass eine 10-jährige Therapie mit Tamoxifen Patientinnen mit Östrogenrezeptor (ER)-positivem Mammakarzinom besser vor weiteren Rezidiven schützt und die Brustkrebssterblichkeit deutlicher reduziert als eine 5-jährige Therapie, scheint sich die Tendenz hin zu einer längeren Verabreichung nun auch für Aromatasehemmer zu bestätigen. Allerdings mit Einschränkungen. Die viel diskutierte MA.17R-Studie, die Paul E. Goss, Boston, in Chicago vorstellte [1] und die parallel auch in der Online-Version des New England Journal of Medicine erschien [2], verglich die Vorteile einer 5-jährigen mit einer 10-jährigen Verabreichung von Letrozol bei Patientinnen mit ER-positivem Mammakarzinom. Es stellte sich heraus, dass sich das 5-Jahres-krankheitsfreie Überleben (DFS) durch die verlängerte Gabe um $4 \%$ verbesserte (91\% vs. 95\%, Hazard Ratio (HR) 0,66, p = 0,01), überwiegend bedingt durch eine Verminderung der Inzidenz kontralateraler Mammakarzinome. Der DFS-Vorteil ging einher mit einer 34\%igen Reduktion der Rezidivrate. In Zahlen: Von 959 Patientinnen in jeder Gruppe ent- wickelten 13 (10 Jahre Letrozol) gegenüber 31 (5 Jahre Letrozol) ein kontralaterales Mammakarzinom und 55 vs. 68 erlitten einen Rückfall der Erkrankung. Die Lebensqualität der Frauen verschlechterte sich unter der verlängerten Letrozolgabe nicht. Allerdings: Der DFS-Vorteil spiegelte sich nicht in einem Vorteil im Gesamtüberleben (OS) wider. Dies ist der Grund, warum unter Experten umstritten ist, ob die verlängerte Gabe eines Aromatasehemmers nun einen neuen Therapiestandard darstellt, zumal unter einer verlängerten Aromatasehemmer-Therapie auch das erhöhte Risiko von Knochenkomplikationen beachtet werden muss. Hier ist sicher das letzte Wort noch nicht gesprochen.

\section{Glioblastom: Besseres Überleben durch Temozolomid plus Radiatio}

Bei älteren Patienten mit einem neu diagnostizierten Glioblastom verbessert die Kombination der Substanz Temozolomid (TMZ) mit einer Kurzzeit-Radiatio das Überleben der Patienten im Vergleich zu einer herkömmlichen Radiatio. Diese hoffnungsvollen Daten stellte James R. Perry, Toronto, beim ASCO vor [3]. Bislang hatten Onkologen bei älteren Patienten evidenzbasiert nur die Wahl zwischen einer alleinigen Strahlentherapie oder einer alleinigen Chemotherapie mit TMZ. In der Studie erhielten 562 Patienten über 65 Jahre randomisiert entweder eine alleinige Strahlentherapie (40 Gy/15 Zyklen/3 Wochen) oder eine auf 12 Zyklen verkürzte Strahlentherapie plus adjuvantes TMZ. Bei den Patienten unter der Kombinationstherapie zeigte sich verbessertes progressonsfreies Überleben (PFS) ebenso wie ein verbessertes OS (HR 0,67; $\mathrm{p}<0,0001)$. Die Patienten mit einer MGMT-PromotorMethylierung profitierten besonders von der Kombination, doch auch die übrigen Patienten hatten einen Benefit. Die Kombinationsbehandlung war nicht mit einer verschlechterten Lebensqualität assoziiert, die Toxizität handhabbar.

\section{Pankreaskarzinom: Kombinationstherapie verbessert Überleben}

Die Phase-III-Studie ESPAC-4 hatte untersucht, ob Patienten mit einem resezierten Pankreaskarzinom von einer Kombinationstherapie aus Gemcitabin und Capecitabin profitieren im Vergleich

\section{KARGER}

Fax +497614520714 
zu einer alleinigen Gemcitabin-Therapie [4]. 722 Patienten mit einem resezierten Pankreaskarzinom erhielten entweder 6 Zyklen Gemcitabin $\left(1000 \mathrm{mg} / \mathrm{m}^{2}, \mathrm{~d} 1,8,15 ; 24\right.$ Wochen) oder die gleiche Therapie, ergänzt durch Capecitabin $\left(1660 \mathrm{mg} / \mathrm{m}^{2} / \mathrm{Tag}, 21 / 28 \mathrm{~d}, 24\right.$ Wochen). Im Anschluss an die Randomisierung erfolgte alle 3 Monate ein Follow-up bis zum Tod der Patienten. Nach 5 Jahren lebten noch $29 \%$ der Patienten, die die neue Kombinationstherapie erhalten hatten, dagegen nur $16 \%$ der Patienten unter alleiniger Gemcitabin-Therapie. Die Toxizität der Kombinationsbehandlung war akzeptabel. Nach Ansicht der Autoren stellt die Kombinationstherapie den neuen Therapiestandard bei Patienten mit reseziertem Pankreaskarzinom dar.

\section{Lungenkarzinom: deutlicher Benefit durch webbasiertes Follow-up}

Eine sehr interessante französische Phase-III-Studie konnte zeigen, dass Patienten mit Lungenkarzinom erheblich von einer webbasierten Applikation profitieren, die regelmäßig ihre Symptome abfragt [5]. Rationale der Untersuchung war, dass Patienten mit Lungenkrebs, die einen Progress erleiden und entsprechende Symptome entwickeln, sich oftmals erst nach mehreren Wochen bei einem Arzt vorstellen, was eine suboptimale Behandlung zur Folge hat. Mit dem webbasierten System Moovcare dokumentiert der Patient wöchentlich seine Symptome auf einem Smartphone, Tablet oder PC. Wenn die Algorithmen des Systems auf Basis der Daten einen möglichen Progress oder Komplikationen identifizieren, werden via E-Mail Pflegekräfte informiert, die dann den Patienten anrufen und zu einem Arztbesuch einladen. Dieses einfache System führt zu einem deutlichen Überlebensvorteil für die Patienten von 7 Monaten (HR $0,33)$ - und das ganz ohne pharmakologische Intervention.

\section{Checkpoint-Inhibitor erfolgreich auch beim Blasenkarzinom}

Die Immuntherapie mit anti-PD-1/PD-L1-Checkpoint-Inhibitoren erlebt einen ungebrochenen Höhenflug. Nachdem die meisten Daten für das maligne Melanom und das nicht-kleinzellige Lungenkarzinom (NSCLC) vorliegen und auch schon erste Zulassungen für diese Indikationen vorliegen, werden die Medikamente derzeit bei mindestens 30 Tumorentitäten getestet, darunter das tripelnegative Mammakarzinom, Kopf-Hals-Tumoren oder das Magenkarzinom. Anders als bei zielgerichteten Therapien, die in der Regel die Tumorkontrolle verbessern und die Progression der Erkrankung hinausschieben, wird unter eine Immuntherapie bei einem nicht unerheblichen Teil der Patienten ein Langzeitüberleben beobachtet. In aktuellen Studien werden Immuntherapeutika auch miteinander oder mit anderen Therapien kombiniert, um die Wirkung zu verbessern und die Nebenwirkungen zu reduzieren.

Atezolizumab ist ein monoklonaler Antikörper, der gegen PDL1, also den Liganden von PD-1 [1] gerichtet ist. Nachdem der Immuncheckpoint-Inhibitor in einer Phase-II-Studie seine Effekti- vität in der Zweitlinie nach Cisplatin-Therapieversagen beim lokal fortgeschrittenen oder metastasierten Harnblasenkarzinom gezeigt hatte [6], untersuchte nun eine einarmige Studie Atezolizumab als Erstlinientherapie bei Patienten mit metastasiertem Blasenkarzinom [7]. Die 119 im metastasierten Setting Chemotherapie-naiven Patienten (mittleres Alter 73 Jahre) waren für eine Chemotherapie mit Cisplatin nicht geeignet. Sie erhielten eine Monotherapie mit 1200 mg Atezolizumab alle 3 Wochen bis zum Krankheitsprogress. Die PD-L1 Expression auf tumorinfiltrierenden Immunzellen wurde immunhistochemisch bestimmt und als IC 0,1 oder 2/3 eingestuft. Eine erste Analyse der Kohorte 1 der IMvigor210-Studie ergab nach einem medianen Follow-up von 14,4 Monaten ein medianes OS von 14,8 Monaten. Die objektive Ansprechrate (ORR) nach RECIST, primärer Wirksamkeitsendpunkt, lag bei $24 \%$, wobei Patienten aus allen IC-Subgruppen und auch Patienten mit schlechten prognostischen Faktoren ansprachen. Das Ansprechen erfolgte schnell und vor allem dauerhaft. Am Stichtag der Analyse sprachen $75 \%$ der analysierten Patienten noch immer auf die Therapie an. Die geschätzte 12-Monats-Gesamtüberlebensrate lag bei $57 \%$. Insgesamt erwies sich Atezolizumab als gut verträglich. Grad 3/4-Nebenwirkungen waren selten. Nun müssen randomisierte Studien zeigen, ob der PD-L1-Inhibitor auch in Kombination mit Chemotherapie seine Vorteile behält und ob die PD1Inhibition bevorzugt in der Erstlinie eingesetzt werden sollte.

\section{Multiples Myelom: Dreidosisregime mit Daratumumab erfolgreich}

Die pivotale Phase-III-Studie CASTOR, die beim ASCO vorgestellt wurde, zeigte, dass Patienten mit rezidiviertem und refraktärem Multiplen Myelom (MM) von einem Behandlungsansatz profitieren, bei dem der anti-CD38-Antikörper Daratumumab (zugelassen als Monotherapie beim mehrfach vorbehandelten MM) zusätzlich zum Zweier-Standerregime Bortezomib und Dexamethason gegeben wird. Rund 500 Patienten hatten randomisiert entweder Bortezomib/Dexamethason alleine oder die Dreikombination erhalten. Primärer Endpunkt war das PFS. Die Daten einer vorspezifizierten Interimsanalyse wurden beim ASCO vorgestellt [8]. Der primäre Endpunkt der Studie wurde erreicht. Das Risiko, einen Progress zu erleiden oder zu versterben war unter der Dreierkombination um $61 \%$ reduziert (HR 0,39). Der Vorteil zeigte sich in allen klinisch relevanten Patienten-Subgruppen - bei einem Sicherheitsprofil, das konsistent mit dem einer Daratumumab-Monotherapie sowie Bortezomib und Dexamethason alleine war. Nach Ansicht der Autoren stellt die Dreierkombination den neuen Therapiestandard beim rezidivierten und refraktären MM dar.

Dr. Claudia Schöllmann, Grasbrunn

\section{Quelle}

Jahrestagung der American Society of Clinical Oncology (ASCO), 3. bis 7. Juni 2016 in Chicago/IL 


\section{Literatur}

1 Goss PE, Ingle JN, et al.: A randomized trial (MA.17R) of extending adjuvant letrozole for 5 years after completing an initial 5 years of aromatase inhibitor therapy alone or preceded by tamoxifen in postmenopausal women with early-stage breast cancer. J Clin Oncol 2016;34 (suppl; abstr LBA1).

2 Goss PE, Ingle JN. et al.: Extending aromatase-inhibitor adjuvant therapy to 10 years. N Engl J Med 2016; [Epub ahead of print] DOI: 10.1056/NEJMoa1604700.

3 Perry JR, Laperriere N, et al.: A phase III randomized controlled trial of short-course radiotherapy with or without concomitant and adjuvant temozolomide in elderly patients with glioblastoma (CCTG CE.6, EORTC 26062-22061, TROG 08.02, NCT00482677). J Clin Oncol 2016;34 (suppl; abstr LBA2).
4 Neoptolemos JP, Palmer D, et al.: ESPAC-4: A multicenter, international, open-label randomized controlled phase III trial of adjuvant combination chemotherapy of gemcitabine (GEM) and capecitabine (CAP) versus monotherapy gemcitabine in patients with resected pancreatic ductal adenocarcinoma. J Clin Oncol 2016;34 (suppl; abstr LBA4006).

5 Denis $\mathrm{F}$, Lethrosne $\mathrm{C}$, et al.: Overall survival in patients with lung cancer using a web-application-guided follow-up compared to standard modalities: results of phase III randomized trial. J Clin Oncol 2016;34 (suppl; abstr LBA9006).

6 Hoffman-Censits JH, Grivas P et al.: IMvigor 210, a phase II trial of atezolizumab (MPDL3280A) in platinum-treated locally advanced or metastatic urothelial carcinoma (mUC). J Clin Oncol 2016;34 (suppl 2S; abstr 355)
7 Vasant Balar A, Galsky MD, et al: Atezolizumab (atezo) as first-line (1L) therapy in cisplatin-ineligible locally advanced/metastatic urothelial carcinoma (mUC): Primary analysis of IMvigor210 cohort 1 . J Clin Oncol 2016;34 (suppl; abstr LBA4500).

8 Palumbo A, Chanan-Khan AAA, et al.: Phase III randomized controlled study of daratumumab, bortezomib, and dexamethasone (DVd) versus bortezomib and dexamethasone $(\mathrm{Vd})$ in patients (pts) with relapsed or refractory multiple myeloma (RRMM): CASTOR study. J Clin Oncol 2016;34 (suppl; abstr LBA4). 